\title{
Cereblon E3 Ubiquitin Ligase Modulating Agent CC-92480
}

National Cancer Institute

\section{Source}

National Cancer Institute. Cereblon E3 Ubiquitin Ligase Modulating Agent CC-92480. NCI

Thesaurus. Code C146660.

A modulator of the E3 ubiquitin lig ase complex containing cereblon (CRL4-CRBN E3 ubiquitin ligase), with potential immunomodulating and antineoplastic activities. Upon administration, cereblon E3 ubiquitin ligase modulating agent CC-92480 specifically binds to cereblon (CRBN), thereby affecting the ubiquitin E3 ligase activity, and targ eting certain substrate proteins for ubiquitination. This induces proteasome-mediated deg radation of certain transcription factors, some of which are transcriptional repressors in T-cells. This leads to modulation of the immune system, including activation of Tlymphocytes, and downregulation of the activity of other proteins, some of which play key roles in the proliferation of certain cancer cell types. CRBN, the substrate recognition component of the CRL4-CRBN E3 ubiquitin ligase complex, plays a key role in the ubiquitination of certain proteins. 